\title{
Relación entre la percepción del clima laboral que tiene el personal de enfermería y el cuidado humano que brinda a la paciente en la Unidad de Cuidados Intensivos Materno del "Instituto Nacional Materno Perinatal", Lima, 2014
}

\author{
Relationship between the perception of the work environment that the nursing staff has and the \\ human care provided to the patient in the Maternal Intensive Care Unit from the National Maternal \\ and Perinatal Institute, Lima
}

Irene Beatriz Espinoza De La Sota ${ }^{1}$, Flor Lucila Contreras Castro²

\begin{abstract}
RESUMEN
Objetivo: Determinar la relación entre la percepción del clima laboral que tiene el personal de Enfermería y el cuidado humano que brinda a la paciente de la Unidad de Cuidados Intensivos Materno (UCIM) del Instituto Nacional Materno Perinatal. Material y métodos: El estudio es de diseño no experimental, transversal y descriptivo correlacional. La población estuvo constituida por 28 profesionales de Enfermería que trabajan en el área de Cuidados Intensivos Materno del Instituto Nacional Materno Perinatal. Para la recolección de datos se aplicó dos instrumentos: un cuestionario para evaluar la percepción del clima laboral, considerando cuatro dimensiones, y una guía de observación para evaluar el cuidado humano; ambos instrumentos fueron elaborados por las investigadoras. Para establecer la relación entre las variables, se utilizó el estadístico de correlación de Rho Spearman. Resultados: Los resultados muestran que no existe relación significativa entre las variables clima laboral y cuidado humano, según la prueba Rho Spearman con $\rho=0.239$, y el valor $\mathrm{p}=0.220$ que es mayor al valor planteado $\alpha=0.05$, sin embargo, se encontró una correlación significativa entre la dimensión implicancia y el cuidado humano que brinda el profesional de Enfermería. Conclusiones: No existe correlación significativa entre el clima laboral percibido por el profesional de Enfermería y el cuidado humano que brinda a la paciente de la Unidad de Cuidados Intensivos Materno (UCIM) del Instituto Nacional Materno Perinatal.
\end{abstract}

Palabras clave: Clima laboral, cuidado humano y enfermería.

\begin{abstract}
Objective: To determine the relationship between the perception of the work environment that has nurses and human care provided to the patient in Intensive Care Unit Maternity (MICU) of the National Maternal and Perinatal Institute. Methods: The study is not experimental, transversal and descriptive correlational design. The population consisted of 28 nurses working in the area of Maternal Intensive Care National Maternal Perinatal Institute. For data collection was applied two instruments: a questionnaire to assess the perception of the work environment by considering four dimensions, and an observation guide for assessing human care; Both instruments were developed by the researcher. Statistical Rho Spearman correlation was used to establish the relationship between the variables. Results: The results show that there is no significant relationship between the variables work environment and human care as tested Rho Spearman $\rho=0.239$, and $p=0.220$ value that is greater than the value set $\alpha=0.05$, however, he found a implication significant correlation between the human dimension and care provided by the nurse. Conclusions: There is no significant correlation between the work environment perceived by the nurse and human care provided to the patient in the Intensive Care Unit Maternity (MICU) of the National Maternal and Perinatal Institute.
\end{abstract}

Keywords: Work environment, human care and nursing. 


\section{INTRODUCCIÓN}

El clima laboral empezó a cobrar importancia entre los investigadores, desde los años sesenta, denominado ambiente, atmósfera, clima laboral, ambiente interno de la organización, entre otras. Asimismo, comienza a tomar fuerza la idea de que en una organización no es únicamente importante la estructura física, sino también el recurso humano que en ella labora e interactúa (Furnham, 2001).

En la actualidad, las empresas e instituciones han demostrado la importancia que tiene el clima laboral y su repercusión en la productividad, debido a la gran parte de tiempo de vida social que los empleados tienen en las empresas, donde desarrollan la actividad laboral, junto a personas de diferentes caracteres, pero con objetivos en común (Córdova, 2010).

Por ello, los empleados deben estar identificados con la institución, poseer autonomía en la toma de sus decisiones, contar con un líder que promueva la participación y, sobre todo, debe existir el espíritu para trabajar en equipo (Crane, citado por Chiang, 2010). Del mismo modo, en el área de salud es importante que exista un clima laboral favorable, sobre todo, en las áreas críticas, para mantener al personal con espíritu positivo y dispuesto a brindar un cuidado con calidez humana.

Cabe resaltar que no siempre la interacción con los compañeros de trabajo es buena, razón por la cual surgen conflictos que llevan a tomar decisiones que afectan la relación laboral, la productividad, así como también la calidad del trabajo y el trato hacia las pacientes (Da Silva, 2002). De allí la importancia de realizar el presente estudio, teniendo como objetivo determinar la relación entre la percepción del clima laboral que tiene el personal de Enfermería y el cuidado humano que brinda a la paciente de la Unidad de Cuidados Intensivos Materno del Instituto Nacional Materno Perinatal.

\section{MATERIAL Y MÉTODOS}

El estudio es de diseño no experimental, transversal y descriptivo correlacional. Se realizó en la Unidad de Cuidados Intensivos Materno del Instituto Nacional Materno Perinatal, ubicado en Jr. Miro Quezada, Cercado de Lima. La población estuvo constituida por 28 profesionales de Enfermería.

Para la recolección de datos, se utilizaron dos instrumentos: un cuestionario para medir la variable clima laboral, considerando las dimensiones de autonomía, consideración, cohesión e implicancia, donde los items fueron evaluados según la escala de Likert. Para medir la variable cuidado humano, se aplicó una guía de observación.

Ambos instrumentos fueron elaborados por las investigadoras y sometidos a juicio de expertos para su validación. Para medir la confiablidad, se aplicó una prueba piloto, obteniendo un coeficiente de alfa de Cronbach de 0.849 para el cuestionario de clima laboral, y el valor de 0.76 de la prueba de Kuder Richarson para la guía de observación sobre cuidado humano.

Los datos obtenidos fueron procesados en el programa Spss v19 y el estadístico utilizado fue la prueba de Spearman (Rho).

\section{RESULTADOS}

Tabla 1

Percepción del clima laboral que tiene el personal de enfermería de la Unidad de Cuidados Intensivos Materno, Lima 2014.

\begin{tabular}{lll}
\hline Percepción del clima laboral & $\mathrm{N}$ & $\%$ \\
\hline Muy favorable & 17 & 60.71 \\
Favorable & 11 & 39.29 \\
Desfavorable & 0 & 0.00 \\
Muy desfavorables & 0 & 0.00 \\
Total & 28 & 100.0 \\
\hline
\end{tabular}

En la tabla 1, se observa que el $60.71 \%$ del personal de Enfermería del Instituto Nacional Materno Perinatal, percibe el clima laboral como muy favorable; el $39.29 \%$ lo percibe como favorable y ninguno lo percibe como desfavorable ni muy desfavorable. 
Relación entre la percepción del clima laboral que tiene el personal de enfermería y el cuidado humano que brinda a la paciente en la Unidad de Cuidados Intensivos Materno del "Instituto Nacional Materno Perinatal”, Lima, 2014

Tabla 2

Cuidado humano que brinda el personal de enfermería a la paciente en la Unidad de Cuidados Intensivos Materno, Lima 2014.

\begin{tabular}{ccc}
\hline Cuidado Humano & N & $\%$ \\
\hline Siempre & 27 & 96.43 \\
A veces & 1 & 3.57 \\
Nunca & 0 & 0.00 \\
Total & 28 & 100.0 \\
\hline
\end{tabular}

En la tabla 2, se observa que un $96.43 \%$ del personal de Enfermería, de la Unidad de Cuidados Intensivos Materno, percibe que siempre brinda cuidado humano a la paciente; el $3.57 \%$ percibe que a veces brinda cuidado humano, y ninguno percibe que nunca lo hace.

Tabla 3

Cuidado humano según dimensiones de la percepción del clima laboral.

\begin{tabular}{lllll}
\hline & & & \multicolumn{2}{c}{ Cuidado humano } \\
\cline { 4 - 5 } & $\begin{array}{l}\text { Índice de } \\
\text { confianza }\end{array}$ & $\begin{array}{l}\text { Nivel de } \\
\text { significancia }\end{array}$ & $\rho$ de Spearman & Sig. Bilateral \\
\hline Autonomía & 0,95 & 0,05 & 0,280 & 0,150 \\
\hline Consideración & 0,95 & 0,05 & 0,314 & 0,103 \\
\hline Cohesión & 0,95 & 0,05 & 0,207 & 0,291 \\
\hline Implicancia & 0,95 & 0,05 & 0,413 & 0,029 \\
\hline Clima laboral & 0,95 & 0,05 & 0,239 & 0,220 \\
\hline
\end{tabular}

La tabla 3 muestra la correlación lineal entre el cuidado humano que brinda el profesional deEnfermería y las dimensiones del clima laboral, encontrándose que existe una relación directa y significativa entre el cuidado humano y la dimensión implicancia $($ Rho $=$ 0.413, $\mathrm{p}=0.029$ ); no se encontró relación significativa para las dimensiones de autonomía ( $\mathrm{Rho}=0.280$, $\mathrm{p}=0.150$ ), consideración (Rho $=0.314, \mathrm{p}=0.103)$ y cohesión $(\mathrm{Rho}=0.207, \mathrm{p}=0.291)$. Todos estos valores fueron evaluados con un $\alpha=0.05$. La parte final de la tabla muestra el valor $\mathrm{Rho}=0.239, \mathrm{p}=0.220$ para el clima laboral correlacionado con el cuidado humano, indicando que no existe una relación significativa entre estas variables por tener un $\mathrm{p}=0.220$ que es mayor al valor planteado $\alpha=0.05$.

\section{DISCUSIÓN}

En la presente investigación, se encontró que más de la mitad, de la muestra de estudio, percibe un clima laboral muy favorable en la Unidad de Cuidados Intensivos Materno, similares a los encontrados por Gámez y Perales (1999), en su estudio “Clima laboral en cuatro unidades críticas de un hospital", donde la mayoría del personal de Enfermería está de acuerdo con las funciones que desempeña, manifiesta que existe buenas relaciones con el equipo de salud y que la ergonomía de las unidades ejercen una influencia significativa en el desarrollo de la actividad laboral. Cortés (2009), también reporta resultados positivos en el estudio realizado en una institución de salud, donde el $78 \%$ del personal refiere sentirse motivado en la tarea que realiza y, sobre todo, que tienen libertad de acción cuando ejecutan su trabajo. Estos resultados demuestran compromiso y sentido de pertenencia del personal con su puesto de trabajo y, por consiguiente, con la institución al percibir un buen clima laboral, pues cuando la calidad de vida laboral, ofrecida por una organización, es percibida como agradable por sus empleados, repercute positivamente en la salud del trabajador e impacta favorablemente en su desempeño y los resultado de la organización (Harte, Schmidt y Hayes, 2002).

Por tanto, es muy importante que las instituciones de salud, sobre todo en las áreas críticas como la Unidad de Cuidados Intensivos Materno, donde se realizó el estudio, se proporcione un ambiente laboral favorable, pues el personal afronta una sobrecarga de estrés debido a la naturaleza del trabajo, la alta exigencia y competencia por las características de los pacientes críticos a quienes se atiende, el esfuerzo 
físico que se realiza, el ruido propio del ambiente y la fatiga. En estas circunstancias, un clima laboral favorable beneficiará no solo al personal de salud, sino también al paciente porque recibirá una atención de calidad a través del trabajo en equipo, una buena comunicación interdisciplinaria, basado en el respeto y reconocimiento mutuo de cada uno de sus integrantes (Fernández, 2002).

Respecto a la variable de cuidado humano, los resultados muestran que casi la totalidad de la muestra siempre brinda cuidado humano a las pacientes en la Unidad de Cuidados Intensivos Materno, datos que concuerdan con los encontrados por Gonzales (2008), en un estudio realizado en el servicio de urgencias de gineco-obstetricia, donde el $73 \%$ de las pacientes siempre percibieron cuidado humanizado por las enfermeras. Asimismo, Ramírez y Parra (2011) encontraron que la mayoría de los pacientes, en una Unidad de Cuidados Intensivos, tienen una percepción positiva sobre el cuidado humano recibido.

Estos resultados son alentadores, porque demuestran el compromiso con la profesión y con el prójimo que tiene el personal de Enfermería quien está más tiempo y más cerca del paciente; toman conciencia del verdadero significado del cuidado humano como la esencia de la Enfermería, la fuente de la conciencia que se manifiesta en el cuidado ofrecido (Dillon y Wright, 1996; Strickland, 1996; Fry Killen y Robinson, 1996). Esta actitud marca la diferencia en la vida de la persona que brinda el cuidado y quien recibe el cuidado (Swanson, citado por Marriner y Raile, 2007).

En cuanto a la correlación de la dimensión implicancia del clima laboral y el cuidado humano que brinda el personal de Enfermería a la paciente de la Unidad de Cuidados Intensivos Materno, se encontró que existe relación significativa. Considerando que la implicancia refleja el grado de compromiso y entrega del empleado hacia su empresa, Crane, (1981) y Arciniega (2002) señalan que los trabajadores que poseen altos niveles de compromiso son aquellos que registran un buen nivel de desempeño, de productividad y bajos índices de ausentismo, como se evidencia en el resultado obtenido en el personal de Enfermería de la Unidad de Cuidados Intensivos Materno, quienes se encuentran muy comprometidos con su trabajo y con la unidad, llegando a considerarlo como parte central de su desarrollo profesional y personal (Robbins, 1999). Esto lleva al personal a convertirse en una fuente de innovación, asumir iniciativas, tener espíritu emprendedor y actuar de manera proactiva (Lagomarsino, 2000). Chopra (1994) menciona que el personal de Enfermería comprometido se siente motivado para brindar cuidado humano que se refleja en una sonrisa, una dulce mirada, un afectuoso contacto, posar una mano sobre la persona en señal de amistad; es decir al demostrar interés y compromiso en apoyar el cuidado de su salud.

Por otro lado, no se encontró relación significativa entre el cuidado humano y el clima laboral en las dimensiones de autonomía, consideración y cohesión, así como para las variables generales de clima laboral y cuidado humano, demostrando que el personal de Enfermería brinda cuidado humano independientemente de cómo perciba el clima laboral, pues su trabajo es guiado por su vocación de servicio y compromiso con la profesión (Ortega, 2009). Por consiguiente, se confirma que el cuidado humano brindado por Enfermería no es de sujeto a objeto, sino de sujeto a sujeto, no es una relación de dominación sino de convivencia, no es intervención sino comunicación e interacción; es un servicio humano que permite promover, manejar y salvaguardar la salud y la vida del paciente, considerándolo como un ser holístico y humano (Restrepo citado por Sánchez, 2005).

\section{Declaración de financiamiento y de conflicto de intereses:}

El estudio fue financiado por los autores, quienes declaran no tener algún tipo de conflicto de interés en la investigación realizada.

\section{Correspondencia:}

Irene Beatriz Espinoza De la Sota Enfermera asistencial del servicio de Cuidados Intensivos Materno del Instituto Materno Perinatal. Jr. Miro Quesada Nro. 941. Lima. Perú.

e-mail: irene_1806@hotmail.com 
Relación entre la percepción del clima laboral que tiene el personal de enfermería y el cuidado humano que brinda a la paciente en la Unidad de Cuidados Intensivos Materno del "Instituto Nacional Materno Perinatal”, Lima, 2014

\section{REFERENCIAS BIBLIOGRÁFICAS}

Arciniega, L. (2002). Compromiso organizacional. México.

Córdova, C. (2010). Un clima laboral óptimo. Recuperado de http://www.climalaboral.com.es/category/climalaboral/page/2/

Cortez, N. (2009). Dimensiones del clima organizacional que prevalecen en el Hospital Dr. Luís F. Nachón de la ciudad de Xalapa. (Tesis de Maestría). Universidad de Veracruz. México.

Chiang, M. y Antonio, J. (2010). Relaciones entre el clima organizacional y la satisfacción laboral. Madrid: RB servicios editoriales, S.L.

Chopra, D. (1994). La curación cuántica. Explorando las fronteras de la medicina mental y corporal. México: Editorial Grijalbo, S.A.

Da Silva, (2002). Los nuevos desafios en la gerencia y teorías de la administración. México: Editorial Thompson.

Dillon y Wright, (1996). El arte y la ciencia del cuidado. Grupo de Cuidado Universidad Nacional de Colombia: Unibiblos.

Fernández. D. (2002). Estrés laboral en los profesionales de enfermería hospitalarios. 45. Recuperado de: scielo. isciii.es/scielo.php?pid=S1695-61412012000200018

Furnham, A. (2001). El comportamiento del individuo en las organizaciones. México: Oxford University Press.

Gámez, M. Santano, A., Matías, S. Roca, S. y Tomey, M. (1999). El estudio clima laboral en cuatro unidades críticas de un hospital, en Madrid. Enfermería Intensiva, 10(3). Recuperado de: http://www.elsevier.es/esrevista-enfermeria-intensiva-142-articulo-estudio-delclima-laboral-cuatro-13008796

Gonzales, J. (2008). Percepción del cuidado humanizado en pacientes que ingresan al servicio de hospitalización de urgencias gineco-obstetricia de un nivel de atención II y III. (Tesis de maestría). Pontificia Universidad Javeriana. Colombia.

Harter, J. Schmidt, F. y Hayes, T. (2002). Business unit, level relationship between Employee satisfaccion, employee engagement, and business outcomes: A meta_analysis. Journal of Applied Psycology, 87.
Lagomarsino, R. (2002). Clima y Compromiso Organizacional. IEEM, (15). Recuperado de: http:// www2.um.edu.uy/ieem/Revista/200312/Revista\%20 IEEM\%20200312-

Marriner, T. y Raile, M. (2007). Modelos y teorías en enfermería. España: Copyright MMVL Mosby.

Ortega, C. (2009). Manual de evaluación de la calidad de servicio de enfermería. México: Editorial médica panamericana.

Pritchard y Karasick, (1980). Dimensiones del clima laboral. Recuperado de: http://www.scribd.com/ doc/22935995/Dimensiones-Del-Clima-Laboral

Ramírez, C. y Parra, M. (2011). Percepción de los comportamientos del cuidado de enfermería en la unidad de cuidados intensivos. (Tesis de Maestría). Universidad Nacional de Colombia. Colombia.

Restrepo, L. (1998). Citado por Sánchez, B. (2005). La investigación y el cuidado en América Latina. Bogotá: Editorial Universidad Nacional de Colombia.

Robbins, S. (1999). The effects of organizational climate on managerial job performance and job satisfaction. México: Prentice Hall.

Sánchez, B. (2004). Dimensión espiritual del cuidado de enfermería en situaciones de cronicidad y muerte Bogotá: Editorial Universidad Nacional de Colombia.

Strickland (1996). Citado por Barrera, L. Pinto, N. El arte y la ciencia del cuidado. Colombia: Editorial Universidad Nacional de Colombia.

Swanson, K. (2009). Nursing as informed caring for the well being of others. The journal of nursing scholarship 24(4), 352-357.

Recibido: $15 / 11 / 2014$ Aceptado: 15/03/2015 


\title{
Estilo de vida y síndrome de burnout en trabajadores de la salud de una clínica privada de Lima
}

\author{
Lifestyle and Burnout's syndrome in the health staff in a private clinic in Lima
}

Karen Sabogal Silvestre¹, Sonia Herrera Frías², Daniel Farfán Rodríguez ${ }^{3}$

\section{RESUMEN}

Objetivo: El objetivo del estudio fue determinar la relación entre estilo de vida y síndrome de burnout en 70 trabajadores de ambos sexos del área de salud de una clínica privada de Lima. Material y métodos: Diseño no experimental y de alcance descriptivo-correlacional, donde se utilizó el cuestionario de Maslach Burnout Inventory y el cuestionario de perfil de estilo de vida (PEPS -I). Resultados: Los resultados indican que no existe relación significativa entre estilo de vida y síndrome de burnout $(x 2=2,859 ; p>0,05)$, con respecto al agotamiento emocional se encontró que no existe relación significativa con el estilo de vida $(\mathrm{r}=-, 104 ; \mathrm{p}>0,05)$, de igual manera con la dimensión despersonalización no se halló relación significativa $(r=, 115 ; p>0,05)$. Conclusiones: Finalmente, se encontró que existe relación significativa entre estilo de vida y la dimensión realización personal $(\mathrm{r}=, 232, \mathrm{p} \leq, 05)$.

Palabras clave: Estilo de vida, síndrome de burnout, personal de la salud.

\begin{abstract}
Objective: The objective of the study was to determine the relation between lifestyle and Burnout's syndrome in 70 workers of both genders of the area of health in a private clinic in Lima. Methods: The study had a non-experimental design and a descriptive-correlational reach, where the "Maslach's burnout Inventory" questionaire was used and the lifestyle profile questionnaire (PEPS -I). Results: The results suggest that there is not a significant relation between lifestyle and burnout's syndrome (x2 2.859; P 0.05), according with the emotional exhaustion that it was found. There is not a significant relation with lifestyle ( $\mathrm{r}-, 104 ; \mathrm{P} 0.05)$, likewise, in the depersonalism dimension there was not a significant relation (r, 115; P 0.05). Conclusions: Finally it was found that there is a significant relation between lifestyle and the personal accomplishment dimension ( $\mathrm{r}, 232$, $\mathrm{p}$, $05)$.
\end{abstract}

Keywords: Lifestyle, burnout syndrome, health staff.

${ }^{1}$ Psicóloga del Colegio Adventista Espinar. Cuzco, Perú.

${ }^{2}$ Psicóloga de Beca 18. Universidad Peruana Unión. Lima, Perú.

${ }^{3}$ Profesor Asociado de la EP de Psicología. Universidad Peruana Unión. Lima, Perú. 


\section{INTRODUCCIÓN}

Las características del entorno laboral como los horarios, las exigencias del puesto del trabajo y la carga laboral, sumado a los hábitos nocivos del trabajador, pueden ocasionar consecuencias físicas y psicológicas que afecten la salud (De Oliveira y Monteiro, 2004). Especialmente en los profesionales de la salud, quienes se ven expuestos a enfermedades físicas, mentales, conflictos sociales, muerte $\mathrm{y}$ violencia, que puede ocasionar síndrome de burnout (Miret y Martínez Larrea, 2010).

Asimismo, Marcos (2012) describe que el síndrome de burnout surge ante la activación de factores estresantes emocionales e interpersonales. Siguiendo la perspectiva de Maslach y Jackson, Sosa (2007) explica que el síndrome de burnout se compone de tres dimensiones: cansancio o agotamiento emocional, despersonalización y realización personal, Consuegra (2010) añade que es una reacción al estrés a largo plazo, más que una sensación inmediata, consecuente del trabajo.

En cuanto al cansancio emocional, Maslach y Jackson (1991) afirmaron que esta dimensión se determina por el surgimiento de sentimientos de debilidad y agotamiento frente a las exigencias del trabajo, además de existir dificultades para hallar alguna fuente de respaldo. Por ello, Cruz y Merino (2008) manifiestan que a nivel cognitivo se presentan pensamientos pesimistas, falta de concentración en los quehaceres laborales e ideas erróneas sobre las propias posibilidades. Y, al manifestarse física y/o psíquicamente, surge la sensación descrita como no poder dar más de sí mismo a los demás (Sosa, 2007). Finalmente, Seisdedos (1997) menciona que al manifestarse esta dimensión, la fuerza o capital emocional se va consumiendo, el profesional pierde su capacidad de entrega a los demás. Maslach (2009) refuerza esta afirmación al explicar que aquellos trabajadores que han desarrollado esta dimensión, carecen de suficiente energía para enfrentar otro día $\mathrm{u}$ otro problema.

Por otro lado, según Maslach (2009, 2010), la dimensión despersonalización describe una respuesta negativa, insensible o excesivamente apática frente a diversos aspectos del trabajo. Se suele desarrollar en respuesta al cansancio emocional, si la gente está trabajando muy intensamente y haciendo demasiadas cosas, comenzarán a apartarse, a reducir lo que están haciendo, derivando en la pérdida de idealismo, por ello el trabajador reducirá su calidad y nivel de desempeño, ya que pasará de tratar de hacer su mejor esfuerzo a hacer solo el mínimo agregándose a esto un trato deshumanizado, así lo afirma Sosa (2007), al mencionar que se desarrolla sentimientos, actitudes y respuestas negativas, distantes y frías hacia otras personas, especialmente hacia los beneficiarios del propio trabajo.

Finalmente, respecto a la dimensión realización personal, Maslach y Jackson (1981) lo describen como "sentimientos de competencia y éxito en el trabajo". Por ello al desarrollar este síndrome esta dimensión se ve afectada, ya que hay pérdida de ideales por la fuerte desmotivación al ver que su desarrollo personal no es exitoso. Cruz y Merino (2008) respaldan esta postura al afirmar que hay una constante evitación de las relaciones interpersonales en el entorno de trabajo, trayendo como consecuencia ausentismo, evitación del trabajo, impuntualidad y la existencia de riesgo de conducta adictiva.

Existen diversos factores que están relacionados con el manejo y desarrollo del síndrome de burnout, uno de esos factores es el estilo de vida que, practicado de manera consistente y mantenida en la vida cotidiana, puede ayudar el mantenimiento de la salud o puede colocar en situación de riesgo para la enfermedad (Gamarra, Rivera, Alcalde y Cabellos, 2010).

Como mencionan Nakaruma, Míguez y Arce (2014) fue Freudenberger quien describió que este síndrome es derivado de la dedicación de una causa, estilo de vida o relación que no aporta el refuerzo esperado por el individuo que lo desarrolla y todo esto conlleva a una sensación de fracaso y que además, según Marcos, (2012) se manifiesta a través de fatiga crónica, ineficacia y negación de lo ocurrido.

Un acercamiento a la noción, de lo que significa el estilo de vida, implica diferencias antagónicas que suponen comportamientos individuales y de grupo, en tanto que a la vez que se sostienen en el tiempo también es sujeto a cambio.

Según Pérez y De Juanas (2013), el estilo de vida se entiende como aquellos comportamientos o conductas de una persona, que para Hernández, Armayones, Boixadós, Pousada, Guillamón y Gómez (2009) lo considera como patrones de comportamientos, compartidos por un grupo social. Estos comportamientos y patrones están comprendidos por hábitos, actitudes, tradiciones de acuerdo a Sanabria (2009), agrega Hernández et al. (2009), estos pueden ser modas o valores dominantes en cada momento.

Por ello, estudios como de Campos, Gómez y Lázaro (2003) explican que el personal de salud 
aunque tienen un vasto conocimiento en promoción de estilos de vida saludable y prevención de enfermedades, parece que no los interiorizan en su diario vivir porque sacrifican su salud en bienestar de los demás. Las autoras afirman que existe una estrecha relación entre la dinámica del personal de salud y su labor intrahospitalaria con sus estilos de vida, relación que repercute en la salud; al respecto añade Marcos (2012), que esto convertiría al personal de salud en un grupo especial de riesgo; ya que concurren factores personales, profesionales e institucionales que son causas potenciales de síndrome. Asimismo, se explica anteriormente que los estilos de vida son factores asociados que pueden favorecer o producir un efecto contrario, frente al síndrome de burnout; además teniendo en cuenta que los hábitos y costumbres que realiza un individuo están orientados a lograr su desarrollo.

Por lo expuesto, el objetivo del estudio es determinar si existe relación entre estilo de vida y síndrome de burnout, en los profesionales de salud de una clínica privada de Lima.

\section{MATERIAL Y MÉTODOS}

\section{Participantes}

Contó con la participación de 70 trabajadores de salud de una clínica privada de Lima, entre las edades de 18 a 65 años que incluyen a: médicos, enfermeras, técnicos en enfermería, obstetras, nutricionistas y técnicos en farmacia. Fueron seleccionados mediante un diseño no probabilístico, asimismo, se solicitó consentimiento informado a todos los participantes.

En la tabla 1 se aprecia que la mayoría de los trabajadores, del sector salud, evaluados son mujeres (83\%). Asimismo, casi la mitad de los evaluados se encuentran entre las edades de 26 a 35 años (47,1\%). Con respecto al estado civil, la mayoría son solteros $(67 \%)$. Por otro lado, el $44 \%$ se encuentran laborando hace un año y, finalmente, el $57 \%$ son de la región costa.

Tabla 1

Variables sociodemográficas de los profesionales de la salud de una clínica privada de Lima, 2014.

\begin{tabular}{|c|c|c|c|}
\hline \multicolumn{2}{|c|}{ Datos Sociodemográficos } & \multirow[t]{2}{*}{$\mathrm{N}$} & \multirow[t]{2}{*}{$\%$} \\
\hline Sexo & & & \\
\hline & Masculino & 12 & $17 \%$ \\
\hline & Femenino & 58 & $83 \%$ \\
\hline \multicolumn{4}{|l|}{ Edad } \\
\hline & $18-25$ & 22 & $31,4 \%$ \\
\hline & $26-35$ & 33 & $47,1 \%$ \\
\hline & 36- a más & 8 & $11,3 \%$ \\
\hline \multicolumn{4}{|c|}{ Estado civil } \\
\hline & Soltero & 47 & $67 \%$ \\
\hline & Casado & 22 & $31 \%$ \\
\hline & Conviviente & 1 & $1 \%$ \\
\hline \multicolumn{4}{|c|}{ Profesión } \\
\hline & Médico & 7 & $10 \%$ \\
\hline & Enfermero & 22 & $31 \%$ \\
\hline & Nutricionista & 9 & $13 \%$ \\
\hline & Técnico en enfermería & 23 & $33 \%$ \\
\hline & Técnico en farmacia & 7 & $10 \%$ \\
\hline & Interno en enfermería & 1 & $1 \%$ \\
\hline & Interno en medicina & 1 & $1 \%$ \\
\hline \multicolumn{4}{|c|}{ Tiempo de trabajo } \\
\hline & Meses a 1 año & 31 & $44 \%$ \\
\hline & $2-5$ años & 29 & $41 \%$ \\
\hline & $6-10$ años & 3 & $4 \%$ \\
\hline & 11- más & 4 & $6 \%$ \\
\hline \multicolumn{4}{|c|}{ Religión } \\
\hline & Adventista & 52 & $74 \%$ \\
\hline & No adventista & 17 & $24 \%$ \\
\hline \multicolumn{4}{|c|}{$\begin{array}{l}\text { Región o País de } \\
\text { procedencia }\end{array}$} \\
\hline & Costa & 40 & 57 \\
\hline & Sierra & 19 & 27 \\
\hline & Selva & 8 & 11 \\
\hline & Otro & 2 & 3 \\
\hline
\end{tabular}




\section{Instrumentos}

Para el estudio, se utilizó el cuestionario de perfil de estilo de vida (PEPS -I) que mide, de forma cuantitativa, el nivel de estilo de vida y está integrado por 48 reactivos tipo Likert, con un patrón de respuesta con cuatro criterios. En el estudio se comprobó las propiedades psicométricas de la escala, con respecto a la fiabilidad global se obtuvo un coeficiente alpha de Cronbach adecuado (,916), asimismo, las dimensiones también presentan coeficientes adecuados que oscilan entre ,683 y ,800. Con respecto a la validez fue validado por cinco expertos en el tema y la validez de constructo se comprobó a través de la correlación r de Pearson, donde las dimensiones están correlacionadas significativamente.

En cuanto al cuestionario de Maslach burnout Inventory, tiene la finalidad de evaluar las tres dimensiones del síndrome de burnout: cansancio emocional, despersonalización y realización personal, consta de 22 preguntas formuladas de manera afirmativa, con respuestas dicotómicas, además, el test considera que el síndrome de burnout como una variable continua que se puede experimentar en diferentes niveles y no como variable dicotómica; asimismo, en el estudio se comprobó las propiedades psicométricas de la escala, con respecto a la fiabilidad global se obtuvo un coeficiente alpha de Cronbach adecuado (,87); asimismo, las dimensiones también presentan coeficientes adecuados que oscilan entre , 69 y ,71. Con respecto a la validez fue validado por cinco expertos en el tema y la validez de constructo se comprobó a través de la correlación $\mathrm{R}$ de Pearson, donde las dimensiones están correlacionadas significativamente.

\section{Análisis estadístico}

Para el análisis de los datos se utilizó el programa estadístico SPSS 20, también se utilizó la prueba estadística kolmogorov smirnov, la prueba de chi cuadrado y, finalmente, el estadístico $r$ de Pearson.

\section{RESULTADOS}

\section{Tabla 2}

Coeficiente de correlación entre estilo de vida y síndrome de burnout de una clínica privada de Lima, 2014.

\begin{tabular}{lccc}
\hline & Valor & Gl & P \\
\hline Chi - cuadrado de Pearson & $2,859^{\mathrm{a}}$ & 6 &, 826 \\
$\mathrm{~N}$ casos válidos & 70 & & \\
\hline
\end{tabular}

Se aprecia, en la tabla 2, el valor de la prueba chi cuadrada calculada para una tabla $2 \times 2$ que hace referencia a la asociación entre estilo de vida (ausencia - presencia) y síndrome de burnout (con burnout - sin sobrecarga). La Tabla muestra que no existe asociación entre estilo de vida y síndrome de burnout, por tanto, no es significativa $(\mathrm{X} 2=2,859 \mathrm{gl}$ $=6, \mathrm{p}>.01)$.

Tabla 3

Estilo de vida y dimensiones del burnout en el personal de salud de una clínica privada de Lima, 2014.

\begin{tabular}{lcc}
\hline & \multicolumn{2}{c}{ Estilo de Vida } \\
\cline { 2 - 3 } & $\mathrm{R}$ & $\mathrm{P}$ \\
\hline Agotamiento emocional &,-104 &, 392 \\
Despersonalización &, 115 &, 342 \\
Realización personal &, 232 &, 053 \\
\hline
\end{tabular}

De igual forma, en la tabla 3 no se encontró relación significativa entre estilo de vida y las dimensión agotamiento emocional $(\mathrm{r}=-, 104, \mathrm{p}>$ $0,05)$, de manera similar ocurre con estilo de vida y la dimensión de despersonalización $(\mathrm{r}=115, \mathrm{p}>$ $0.05)$, sin embargo, se aprecia que existe relación significativa entre el grado de realización personal y estilo de vida $(\mathrm{r}=, 232, \mathrm{p}<0,05)$. 


\section{DISCUSIÓN}

Si bien la proporción de trabajadores evaluados, que sufren síndrome de burnout (43\%), coincide con la tendencia observada en otras investigaciones (Mera y More, 2014 \& Braga, et al, 2007), la mayoría del personal de salud evaluado mantiene un estilo de vida saludable, siendo la religión un factor importante en la práctica de conductas promotoras de salud, se afirma que tener creencias religiosas es un reforzador permanente que ejerce influencia en el desarrollo de un estilo de vida saludable (Carreño Del Valle, Cladellas \& Ivanovic, 2011). Este factor tambien podría ser un factor interviniente en el desarrolllo de estilos de vida saludables, siendo que la mayoría de los participantes señalaron ser adventistas.

En el estudio se encontró que no existe asociación significativa entre el estilo de vida y el síndrome de burnout ( $\mathrm{x} 2=2,859 ; \mathrm{p}>0,05)$ en el personal de salud de una clínica privada, este resultado no coincide con diferentes estudios que señalan que sí existe correlación inversa entre estilo de vida y síndrome de burnout (Marcos, 2012 \& Simões Moura, 2014, esto hace pensar que existen otras variables que están causando el síndrome de burnout, entre ellas la sobrecarga laboral, la rotación de turno, la familia y el rol (Vergel, Orozco y Ospina, 2013). En relación a ello Gomero et al. (2005) afirman que la insatisfacción laboral es un predictor para el desarrollo del síndrome de burnout, este factor explicaría la presencia de síndrome de burnout, ya que más de la mitad de los trabajadores $(61 \%)$ presentan baja realización personal. De manera similar González y Pérez (2012) mencionan que existe una correlación negativa entre satisfacción laboral, motivación y síndrome de burnout, a ello Barrios, Arechabala y Valenzuela (2012) añaden que el exceso de trabajo, laborar más de 9 horas consecutivas y atender, por lo menos, a cinco pacientes simultáneamente modulaba la respuesta ante el estrés, ocasionando agotamiento emocional, despersonalización y baja satisfacción.

En el estudio también se evidencia que no existe relación significativa entre las dimensiones de agotamiento emocional y despersonalización con el estilo de vida, datos que difieren de las investigaciones (Marcos, 2012 \& Simões Moura, 2014), quienes señalan que hay una relación inversa entre estas dimensiones y el estilo de vida. Cabe mencionar que la despersonalización es la consecuencia directa del agotamiento emocional. Es decir, la conducta distante, fría y desinteresada hacia los pacientes sería resultado del agotamiento emocional, de la sensación de no poder contar con los recursos y así poder manejar la situación.
Arias, Masías y Justo (2014) mencionan que tanto la despersonalización como el agotamiento emocional estarían relacionados con rasgos de personalidad, de modo que las características personales juegan un rol importante en la manifestación del síndrome. Este factor también fue observado por Roth y Pinto (2010), quienes manifiestan que la personalidad, específicamente el neuroticismo, estaría relacionado positivamente con el agotamiento emocional y la despersonalización, y se asocia negativamente con la satisfacción laboral.

Por su parte, Preciado (2007) coincide al señalar que además del estilo de vida, la forma cómo se percibe el trabajo y las características del trabajo sería un factor que desencadena el agotamiento emocional y, por ende, la despersonalización.

Respecto a estilo de vida y la dimensión realización personal, se demuestra que sí existe relación, es decir, las personas que manifiestan tener un mejor estilo de vida también sienten tener mayores logros personales y autoeficacia en el trabajo, como lo señala Amaya y Carrillo (2006). De igual manera, un estilo de vida saludable juega un papel importante para desempeño laboral y realización personal, conformándose así un adecuado bienestar psicológico (García y Gonzáles, 2000).

\section{Declaración de financiamiento y de conflicto de intereses:}

El estudio fue financiado por los autores, quienes declaran no tener algún tipo de conflicto de interés en la investigación realizada.

\section{Correspondencia:}

\section{Karen Sabogal Silvestre}

Psicóloga del Colegio Adventista Espinar. Avenida Ricardo Palma 209 - Espinar, Cusco-Perú.

e-mail: pskarensabogal@gmail.com 


\section{REFERENCIAS BIBLIOGRÁFICAS}

Arias, W., Justo, O., y Masias, A. (2014). Felicidad, síndrome de burnout y estilos de afrontamiento en una empresa privada. Avances en Psicología, 2 (22), 75-87. Recuperado de: http://www.unife.edu.pe/publicaciones/ revistas/psicologia/2014/AVANCES.W.Arias.pdf.

Campos, G., Campos, A., Gómez, C., y Lázaro, K. (2003). Factores personales, organizacionales, sociales y del ambiente laboral que intervienen en la salud del personal de enfermería del hospital San Vicente de Paúl en heredia. un enfoque de investigación - acción. seminario de graduación, práctica dirigida. (Tesis de Licenciatura en Enfermería). Universidad de Costa Rica, Costa Rica.

Carreño. J., Del Valle, C., Cladellas, R., e Ivannovic, D. (2011). La comunicación para la salud y sus aportes desde el modelo de Pender: comunicación y cultura religiosa. Revista de comunicación de la SEECI, 26(11), 22-1. Recuperado dehttp://www.seeci.net/revista/ hemeroteca/Numeros/NUMERO26/Varios2.pdf.

Carillo, H., y Amaya, M. (2006). Estilo de vida saludables de las enfermeras (os) docentes de la Escuela de Enfermería de la Universidad Pedagógica y Tecnológica de Colombia. (Tesis para optar por el grado de licenciatura en enfermería). Universidad Pedagógica y Tecnológica de Colombia, facultad de ciencias de la salud. Recuperado de: http://www.index-f.com/ lascasas/documentos/lc0128.pdf

Consuegra, N. (2010). Diccionario de psicología. Bogotá: Ecoe Ediciones.

Cruz, M. y Merino, J. (2008). Burnout en profesionales de enfermería que trabajan en centros asistenciales de la octava región, Chile. Ciencia y Enfermería, 14 (2), 75-85. Recuperado de http://www.scielo.cl/pdf/cienf/ v14n2/art10.pdf.

De Oliveira, V., y Monteiro, M. (2004). O Estilo de vida dos docentes de enfermagem de um centro universitário. Redalyc, 1 (4), 15-17. Recuperado de http://www. redalyc.org/articulo.oa?id=84226084003.

Gamarra, M., Rivera, H., Alcalde, M., y Cabellos, D. (2010). Estilo de vida, autoestima y apoyo social en estudiantes de enfermería. Scientia, 2 (2), 34-36. Recuperado de http://revistas.concytec.gob.pe/pdf/ scientia/v2n2/a08v2n2.pdf

García, C., y Gonzáles, I. (2006). La categoría bienestar psicológico. Su relación con otras categorías sociales. Revista Cubana de medicina general integral, 16 (6), 586-591. Recuperado de: http://scielo.sld.cu/scielo. php?pid=S0864-21252000000600010\&script $=$ sci arttext.

González, M. (2008). El Síndrome de agotamiento profesional en oncología. Madrid: Editorial Médico Panamericana. Recuperado de: http://books.google. com.pe/books?id=cAwW8nUg 0YC\&pg=PA1\&dq=hi storia+burnout\&hl=es-419\&sa $=$ X\&ei $=$ klZGVM-5Doj
PggSr5IG4Bw\&ved=0CBoQ6AEwAA\#v=onepage \&q $=$ historia $\% 20$ burnout $\& \mathrm{f}=$ false .

Gonzáles, E., y Pérez, E. (2012). Condiciones laborales y desgaste profesional en trabajadores de la salud. Alternativas en Psicología 16 (27), 8-21. Recuperado de http://alternativas.me/attachments/article/4/1.\%20 Condiciones $\% 201$ aborales $\% 20 y \% 20$ desgaste $\% 20$ profesional $\% 20$ en $\% 20$ trabajadores $\% 20 \mathrm{de} \% 201 \mathrm{a} \% 20$ salud $\sim$.pdf

Gomero, R., Palomino, J., Ruiz, F., y Llap. (2005). El Síndrome de burnout en personal sanitario de Los hospitales de la empresa minera de Southern Perú Copper Corporation: Estudio Piloto. RevMedHered, 16 (4), pp.233-238. Recuperado de: http://www.scielo. org.pe/scielo.php?script $=$ sci_arttext\&pid $=\mathrm{S} 1018$ 130X2005000400002

Hernández, E., Armayones, M., Boixadós, M., Pousada, M., Guillamón, N., y Gómez, B. (2009). Salud y Red. Barcelona: Editorial UOC. Recuperado de: http:// books.google.com.pe/books?id=iALqRJToteIC\&pg= PA55\&dq=definici $\% \mathrm{C} 3 \% \mathrm{~B} 3 \mathrm{n}+\mathrm{de}+$ estilos + de + vida + saludable \&hl=es-419\&sa $=$ X\&ei $=$ hUhGVJiLLZLKg gTYkIKoAw\&ved=0CCMQ6AEwAA\#v=onepage\&$\mathrm{q}=$ definici $\% \mathrm{C} 3 \% \mathrm{~B} 3 \mathrm{n} \% 20 \mathrm{de} \% 20$ estilos $\% 20 \mathrm{de} \% 20$ vida $\% 20$ saludable $\& \mathrm{f}=$ false.

Marcos, C. (2012). Estilos de vida y Síndrome de Burnout en profesionales de enfermería de Hospital Guillermo Almenara Irigoyen Marzo, 2012. Recuperado de: http:// cybertesis.urp.edu.pe/handle/urp/284.

Maslach, Ch. Jackson, S. (1981) The measurement of experienced burnout. Journal of occupational behavior 2 (99).

Maslach, C. y Jackson, S. (1985). The Role of Sex and Family Variables in Burnout. Sex Roles, 12 (7), 837851.

Maslach, C. (2009). Comprendiendo el Burnout. Revista Ciencia y Trabajo, 32, 37-43. Recuperado de http:// www.cienciaytrabajo.cl/pdfs/32/ pagina37.pdf.

Maslach, C. (2010).The Client Role in Staff Burn-Out. Journal of Social Issues, 34 (4), 111-124. Recuperado de http://onlinelibrary.wiley.com/doi/10 .1111/josi.197 8.34.issue-4/issuetoc.

Miret, C., y Martínez Larrea, A, (2010). El profesional en urgencias y emergencias: agresividad y burnout. Anales del sistema sanitario de navarra, 33, 193-201. Doi: 10.4321/S1137-66272010000200019.

Pérez, G., y De Juanas, A. (2013). Calidad de vida en personas adultas mayores. Madrid: Publicaciones Universidad de educación a distancia. Recuperado de: http://books.google.com.pe/ books?id $=16 \mathrm{VqTMbteRcC} \& p g=\mathrm{PA} 235 \& \mathrm{dq}=$ origenes + de+estilos+de+vida\&hl=es419\&sa=X\&ei=PjRGVM2K GJLNggS05oG4Cg\&ved $=0$ CCoQ6AEwAw\# $\mathrm{v}=$ onepag e \& $q=$ origenes $\% 20$ de $\% 20$ estilos $\% 20$ e $\% 20$ vida $\& \mathrm{f}=$ false. 
Nakaruma, A., Míguez, C., y Arce R. (2014). Equilibrio psicológico y burnout académico. Revista de Investigación, 12 (1), p. 32-39. In: 1697-5200. Recuperado de: http: //webs.uvigo.es/reined/.

Sanabria, F., y Alfonso, P. (2009). Estilos de vida saludable en profesionales de la salud colombianos. Red Revista Med, 15 (2), 208-209. Recuperado de http://site.ebrary. com/lib/bupeusp/Doc?id=10311395\&ppg=4.

Seisdedos, N. (1997). Manual MBl, Inventario Burnout de Maslach. Madrid: TEA.

Sosa, E. (2007). Frecuencia de los síntomas del sindorme de Burnout en profesionales Médicos. Revistas Medica Rosario, 73(20), p. 12-20.Recuperado de: http://www.
planetadoctor.com/documentos/BURNOUT/sintomarg-rosario.pdf.

Preciado, M., Vázquez,J., Pando, M., Gutiérrez, F., Lira, A., Pérez González, M., y González, P. (2007). Validez factorial de la "Escala de agotamiento emocional laboral" para médicos de instituciones públicas en Guadalajara. Redalyc, 9 (1), 41-48. Recuperado de: http://www.redalyc.org/pdf/142/14290107.pdf

Roth, E., y Pinto, B. (2010). Síndrome de Burnout, Personalidad y Satisfacción Laboral en Enfermeras de la Ciudad de La Paz. Ajayu, 8 (2), 62-99. Recuperado de: http://www.ucb.edu.bo/publicaciones/ajayu/v8n2/ v8n2a4.pdf.

Recibido: 18/01/2015

Aceptado: 28/05/2015 